\section{The effects of carotid endarterectomy on ocular haemodynamics}

YEW MENG WONG, JOHN B. CLARK, IRWIN B. FARIS, COLIN B. STYLES, JOSEPH A. KISS
Many techniques have been developed for the measurement of ocular blood flow and velocity. Each has significant disadvantages when used on human subjects and has therefore a limited place in clinical settings. ${ }^{3}$ Erickson et al. in 1989 were the first to report the application of colour Doppler ultrasound (CDU) imaging to retrobulbar vessels. ${ }^{4}$ Since then there has been increasing interest in its use and several groups have been able to confirm its practicability and adequate reproducibility in selected vessels. $^{5-9}$

Colour Doppler analysis of the ophthalmic circulation in the presence of atherosclerotic carotid disease has been the subject of studies by Wolfgang, $\mathrm{Hu}$ and their respective collaborators. ${ }^{10,11}$ Wolfgang et al. looked at one patient and concluded that CDU findings correlated with the more established modalities, namely fluorescein angiography and ophthalmoplethysmography. Hu's work suggested that an inverse relationship exists between ophthalmic and central retinal artery velocities and the degree of carotid artery stenosis.

Our study was directed at characterising changes in the velocities and resistive indices of selected retro-orbital vessels following carotid endarterectomy. We also sought correlations between velocity measurements by CDU and ophthalmodynamometry readings, as well as intraoperative internal carotid arterial stump pressures. A component of this investigation compared the reproducibility of ocular CDU between normal healthy volunteers and older vasculopathic patients. ultrasound, Endarterectomy

The relationship between atherosclerotic carotid disease and conditions of ophthalmic vascular insufficiency has been recognised since Fisher in 1952 described a short series of patients who had suffered strokes following transient monocular blindness. ${ }^{1}$ Amaurosis fugax resulting from a thrombo-embolic event in the eye is a common presenting complaint among patients with carotid disease. More severe but less frequent is the problem of hypotensive retinopathy and the ocular ischaemic syndrome. $^{2}$

\section{Methods}

Prior approval was obtained from the Geelong Hospital Research and Ethics Advisory Committee. Every participant provided written informed consent after full explanation by the principal investigator.

A preliminary study was undertaken to establish the reproducibility of ocular CDU. Twenty healthy volunteers were recruited from among the hospital staff (age 23-60 years, median 26 years; 6 males). In 10 subjects the ophthalmic artery (OA) and central retinal

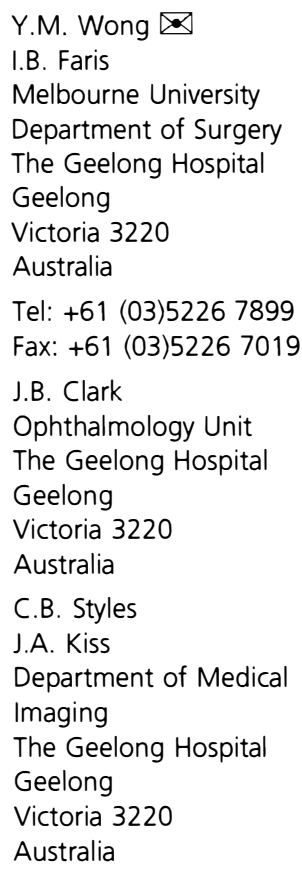

This project was supported by the Geelong Region Medical Research

Foundation 


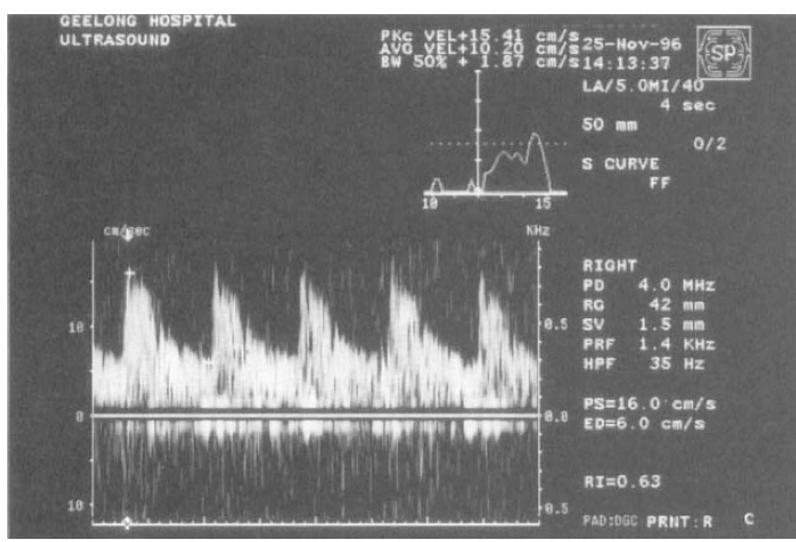

Fig. 1. Spectral analysis of ophthalmic artery on the operated side. Pre-operative peak systolic velocity (PSV) was $16 \mathrm{~cm} / \mathrm{s}$.

artery (CRA) were assessed, while in the remainder the central retinal vein (CRV) and the nasal (NPCA) and temporal (TPCA) posterior ciliary arteries were examined. All examinations were performed in the supine position. A brief medical and ophthalmic history was taken and vital signs recorded prior to each scan, since marked fluctuations in systemic blood pressure have an effect on ocular blood flow velocities. ${ }^{12,13}$ The brachial blood pressure and radial pulse rate were checked for variability of less than $20 \%$ between the first and second measurements.

A Diasonics Master Series colour Doppler scanner (Diasonics, USA) with a $5 \mathrm{MHz}$ linear array transducer placed lightly on the closed upper eyelid was utilised. Coupling gel was applied to optimise contact. The relevant vessel was located and spectral analysis acquired with a $1.5 \mathrm{~mm}$ sample gate $(1.2 \mathrm{~mm}$ when applied to PCA) after correcting for the Doppler angle where necessary. For PCAs, this angle was assumed to be $0^{\circ}$ since we were unable to image an adequate length of these vessels. The OA was studied immediately after it crossed the optic nerve on its medial side, CRA and CRV 2-4 mm posterior to the optic disc within the substance of the optic nerve, and NPCA and TPCA within $5 \mathrm{~mm}$ of the posterior wall of the globe on the nasal and temporal sides of the optic nerve respectively. Both eyes were assessed in succession over $30 \mathrm{~min}$. This examination was repeated 22-26 h later with reference to the initial scan in order to best approximate the site of measurement and direction of gaze.

Mean values of the peak systolic velocity (PSV) (Fig. $1)$, end-diastolic velocity (EDV), resistive index (RI), maximum venous velocity $\left(\mathrm{V}_{\max }\right)$, minimum venous velocity $\left(\mathrm{V}_{\min }\right)$ and venous pulsitility index $(\mathrm{VPI})$ were derived from three waveforms in each vessel. The RI was defined by RI $=(P S V-E D V) / P S V$ and the VPI by $\mathrm{VPI}=\left(\mathrm{V}_{\max }-\mathrm{V}_{\min }\right) / \mathrm{V}_{\max }{ }^{12}$ The coefficient of variation (CV) of those three parameters for all five vessels was calculated based on the equation: $\mathrm{CV}=$ (standard deviation of the mean difference/grand mean) $100 \%$.

Subjects for the main body of the study were recruited from patients referred to the vascular surgery unit and ultrasound department. A total of 38 patients ( 27 men, 11 women; age range 53-83 years), consented to participate of whom 15 were being treated conservatively and classified as the non-operative group. Only one of these patients had symptoms of cerebral ischaemia. The remaining 23 subjects proceeded to unilateral internal carotid artery endarterectomy and one to a common carotid endarterectomy. There were three exclusions from this group. The first withdrew following a postendarterectomy wound infection, a second patient had a more than $20 \%$ decrease in blood pressure on the followup visit, and the final subject was excluded after a vessel was misidentified as the OA on the pre-operative scan. Of the 20 patients 9 were asymptomatic, 4 presented with amaurosis fugax and 7 with transient ischaemic attacks.

Each subject in the non-operative group had two ocular CDU scans performed 7 (mean) weeks apart. A protocol similar to that described above was applied on both visits. Every subject had the velocities in the OA, CRA and CRV measured and, in addition, seven had the NPCA assessed. TPCA was excluded as it was the least reproducible in our hands. There was a median of 9 weeks between the carotid duplex scan, which detected an average stenosis of $75 \%$, and the initial ocular scans. In addition, an ophthalmic examination was conducted routinely. Visual acuity was determined with a Snellen chart at $6 \mathrm{~m}$ and intraocular pressures measured using a Goldmann applanation tonometer. Fundoscopic examination, and estimation of diastolic CRA pressure by hand-held Bailliart ophthalmodynamometer (Inami, Japan) were performed by an observer who was masked to the results of both carotid and ocular ultrasound. CRA pressure readings were reported as the mean of three measurements. Pupillary dilatation was achieved with $1 \%$ tropicamide drops and corneal anaesthesia with $0.4 \%$ oxybuprocaine. Automated perimetry (Octopus, Interzeag, Switzerland) and fundal photography (Topcon, Japan) were also included. However, only tonometry and ophthalmodynamometry were repeated at the second visit. In three patients the CRA pressures could not be determined with confidence on the second visit. A further three did not have measurements taken on the initial appointments. Of the 24 visits with reportable CRA pressures, 20 were performed in conjunction with ocular blood flow velocity recordings.

All operative subjects had had carotid duplex scans on entry to the study with an average stenosis of $81 \%$ and a range of $50-95 \%$ on the operated side. Doppler grading of carotid stenoses (Table 1) was based on criteria of

Table 1. Carotid duplex ultrasound grading of carotid stenosis

\begin{tabular}{cc}
\hline PSV $(\mathrm{cm} / \mathrm{s})$ & Stenosis $(\%)$ \\
\hline$<120$ & $30+$ \\
$120-150$ & $50+$ \\
$150-170$ & $60+$ \\
$170-220$ & $60-70$ \\
250 & $70+$ \\
$250-290$ & $70-80$ \\
$>300$ & $80+$ \\
Systolic/diastolic blurring & $90+$
\end{tabular}

PSV, peak systolic velocity. 
Table 2. Coefficient of variation (\%) for PSV, EDV, RI and VPI in normal subjects

\begin{tabular}{lccrr}
\hline & PSV $(\mathrm{cm} / \mathrm{s})$ & EDV $(\mathrm{cm} / \mathrm{s})$ & RI & VPI \\
\hline OA & 7.0 & 16.4 & 4.0 & 6.8 \\
CRA & 10.3 & 13.3 & 12.3 & - \\
TPCA & 14.8 & 19.8 & 6.8 & - \\
NPCA & 11.5 & 14.4 & - & 9.3 \\
CRV & 10.8 & 11.0 & \\
\hline
\end{tabular}

PSV, peak systolic velocity; EDV, end-diastolic velocity; RI, resistive index; VPI, venous pulsatility index; OA, ophthalmic artery; CRA, central retinal artery; TPCA, temporal posterior ciliary artery; NPCA, nasal posterior ciliary artery; CRV, central retinal vein.

Gerraty et al. ${ }^{14}$ The time span between the pre-operative carotid duplex and ocular scans ranged from 1 to 39 weeks (median 12 weeks). In 16 patients ocular CDU was done within a fortnight before surgery. Post-operative readings were obtained an average of 6 weeks following the procedure. One patient had had a vitrectomy for phacolytic vitritis $2 \frac{1}{2}$ weeks prior to the second assessment. Every subject had the velocities in the OA (Fig. 2), CRA and CRV measured and, in addition, 13 had the NPCA assessed. Ophthalmic assessment as described above was also conducted. Intraoperative internal carotid artery systolic pressures were determined after the arteries had been exposed and the external and common carotid arteries clamped. A $21 \mathrm{G}$ butterfly needle connected to a transducer was then inserted into the common carotid artery distal to the clamp.

\section{Results}

\section{Normal subjects}

The most reproducible criteria in the normal subjects were the RI and PSV in all vessels except the TPCA (Table 2). In the remaining four vessels, the PSV and RI coefficients were close to or less than the arbitrary $10 \%$ threshold. Every vessel was detectable in these participants.

\section{Non-operative vascular patients}

Reproducibility in the group of non-operative vascular patients is shown in Table 3. The RI was again the most reproducible parameter, especially when derived from arterial measurements. However, the PSV was only

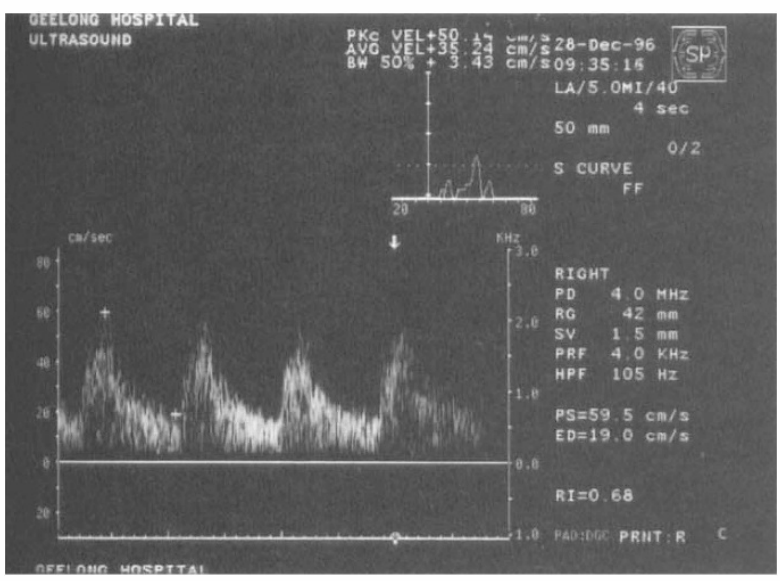

Fig. 2. Spectral analysis of ophthalmic artery on the operated side. Post-operative PSV was $59.5 \mathrm{~cm} / \mathrm{s}$. moderately reproducible, with CV approximating 20\%, 2-3 times that of healthy subjects. Calculations for EDV revealed smaller increases when compared with the first group. We were unable to obtain measurements of the NPCA in two of the seven patients in whom this was attempted.

\section{Correlations between measurements}

Eleven non-operative and 15 pre-operative subjects were examined for a correlation between pressure readings by ophthalmodynamometry and OA, CRA and CRV velocities in a total of 52 eyes and NPCA in 27 eyes. Spearman's rank correlation demonstrated a significant relationship between ophthalmodynamometry and only one parameter: the $\mathrm{V}_{\max }$ in the CRV $\left(r_{\mathrm{S}}=-0.293\right.$; $p<0.05)$.

\section{Operative vascular patients}

There was no significant correlation between systolic intraoperative internal carotid artery stump pressures and pre-operative CDU parameters in 18 operative vascular patients.

On the operated side, the median PSV in every vessel studied rose following surgery (Table 4 ) but was statistically significant only in the OA (Wilcoxon statistic $=186.0 ; p=0.003$ ), as depicted in Fig. 3. No significant change was detected in the contralateral ocular vessels (Fig. 4) nor in the EDV on either side.

There was a significant increase in the post-operative RI of the OA (Wilcoxon statistic $=166.5 ; p=0.023$ ), CRA $($ Wilcoxon statistic $=185.0 ; p=0.003)$ and NPCA (Wilcoxon statistic $=28.0 ; p=0.022$ ) on the endarterectomised side (Figs. 5-7). As for the other parameters, no changes were seen on the opposite side.

There were minor elevations in the CRA perfusion pressure on both sides after surgery, neither of which was statistically significant (Table 5). Measurements were performed on 16 patients but a pre-operative measurement in one eye (non-operated side) was not possible due to active vitritis.

\section{Discussion}

The preliminary study involving healthy normal subjects established that it is possible to achieve adequately reproducible results, in especially the RI and PSV. Consistent with other studies we had most difficulty 
Table 3. Range, mean, standard deviation (SD) and coefficient of variation (CV) for PSV, EDV and RI in non-operative vascular patients

\begin{tabular}{|c|c|c|c|c|}
\hline & Range $(\mathrm{cm} / \mathrm{s})$ & Mean $(\mathrm{cm} / \mathrm{s})$ & $\mathrm{SD}(\mathrm{cm} / \mathrm{s})$ & $\mathrm{CV}(\%)$ \\
\hline \multicolumn{5}{|l|}{ PSV } \\
\hline OA & $14.97-68.13$ & 32.55 & 11.56 & 20.30 \\
\hline CRA & $4.07-36.4$ & 13.05 & 6.12 & 18.44 \\
\hline NPCA & $6.43-24.23$ & 12.31 & 3.97 & 16.26 \\
\hline $\mathrm{CRV}\left(\mathrm{V}_{\max }\right)$ & $4.27-18.3$ & 7.55 & 2.49 & 21.38 \\
\hline \multicolumn{5}{|l|}{ EDV } \\
\hline OA & $3.13-28.57$ & 7.72 & 5.02 & 28.58 \\
\hline CRA & $1.83-8.1$ & 3.73 & 1.43 & 15.18 \\
\hline NPCA & $2.05-6.03$ & 3.41 & 0.91 & 18.73 \\
\hline $\mathrm{CRV}\left(\mathrm{V}_{\min }\right)$ & $3.1-7.53$ & 4.52 & 1.05 & 15.05 \\
\hline \multicolumn{5}{|l|}{ RI } \\
\hline OA & $0.45-0.87$ & 0.76 & 0.09 & 3.23 \\
\hline CRA & $0.46-0.84$ & 0.69 & 0.08 & 3.47 \\
\hline NPCA & $0.57-0.78$ & 0.72 & 0.05 & 4.15 \\
\hline CRV (VPI) & $0.16-0.6$ & 0.38 & 0.09 & 12.85 \\
\hline
\end{tabular}

Abbreviations as in Table 2.

with the PCAs due to the anatomical characteristics of this group of vessels. 6 , ${ }^{6}$ However, a CV of $6.8 \%$ for the NPCA resistive index suggests that attempts at measurements in these arteries may provide useful data. The marked difference in reproducibility between the NPCA and TPCA may be explained by the fact that the majority and largest short PCAs pierce the sclera on the temporal side of the optic nerve ${ }^{15}$ Greenfield and associates noted that there was no significant difference between velocities in the NPCA and TPCA. ${ }^{13}$ Therefore, for subsequent stages in our study, we opted to look at the NPCA as it had the better reproducibility. The EDV was generally less reproducible and this concurs with findings by Baxter and Williamson. ${ }^{6}$ It may be due to the technical difficulty in distinguishing the trough of the waveform from background noise, especially at lower velocities.

Vasculopathic patients were a more difficult population to investigate with CDU. The PSV and EDV had increased CV but the reproducibility of arterial RIs was conserved. This suggests that Doppler angle errors, which influence PSV and EDV but not the RI, are a major factor. Such errors probably occur because of variation in the transducer's position from test to test. ${ }^{9}$ It is virtually impossible to re-create the relative positions of a handheld probe in three-dimensional space and an eye which is free to move. Baxter and Williamson hypothesised that the presence of arteriopathic disease in the posterior ciliary arteries would lead to unsatisfactory spectral analysis in spite of adequate colour flow identification of the vessels. ${ }^{16}$ The inability to detect NPCA in some patients may be due to stenotic lesions reducing blood

Table 4. Median of PSV $(\mathrm{cm} / \mathrm{s})$ before and after surgery on the operated side

\begin{tabular}{lcc}
\hline & Pre-operative & Post-operative \\
\hline OA & 23.88 & 36.68 \\
CRA & 9.92 & 12.32 \\
NPCA & 9.83 & 10.50 \\
CRV & 6.72 & 6.87 \\
\hline
\end{tabular}

Abbreviations as in Table 2. flow beyond the sensitivity of current CDU equipment. However, in the subgroup of patients who underwent surgery, the mean pre- and post-operative PSVs on the non-operated side remained the same. The variation appeared to be less than in the reproducibility studies. There was little difference in the mean velocities between this group and the healthy participants in our study and that of Greenfield et al. ${ }^{13}$ The RI was higher in the CRA, NPCA and CRV.

An isolated negative correlation between diastolic perfusion pressures in the CRA as measured by ophthalmodynamometry and maximum CRV velocities was an unexpected result. If it is true that CRV velocities provide a better indication of retinal perfusion, then this would suggest that an increase in pressure does not

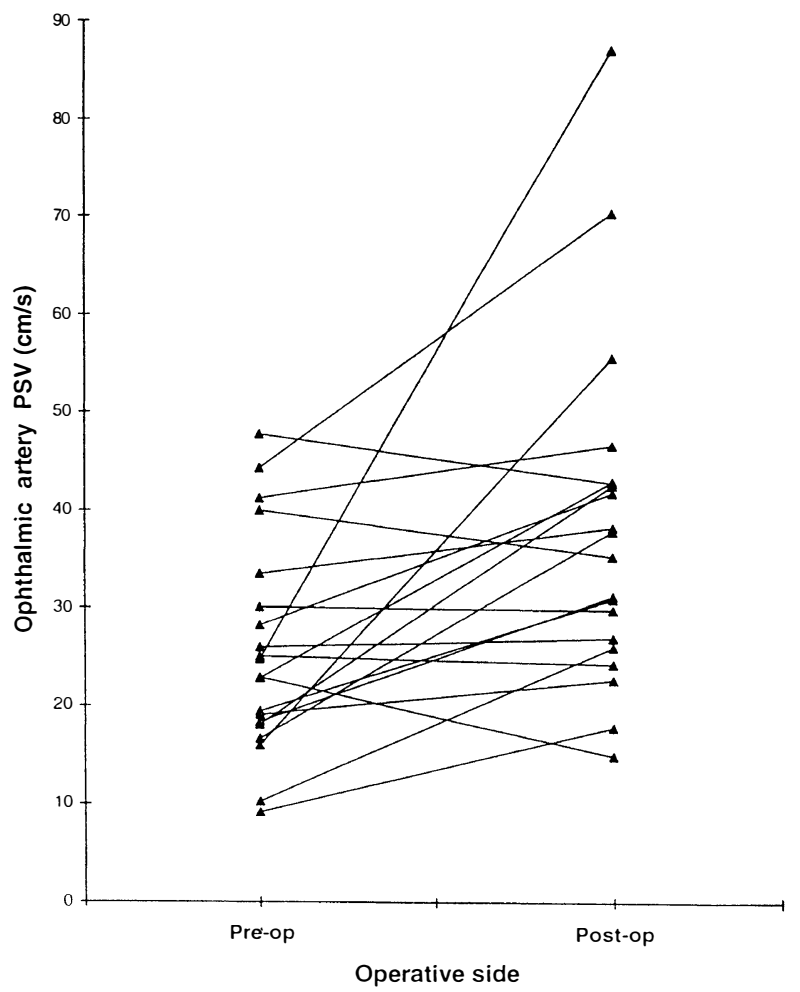

Fig. 3. Changes in PSV in the ophthalmic artery on the operated side before and after surgery. 


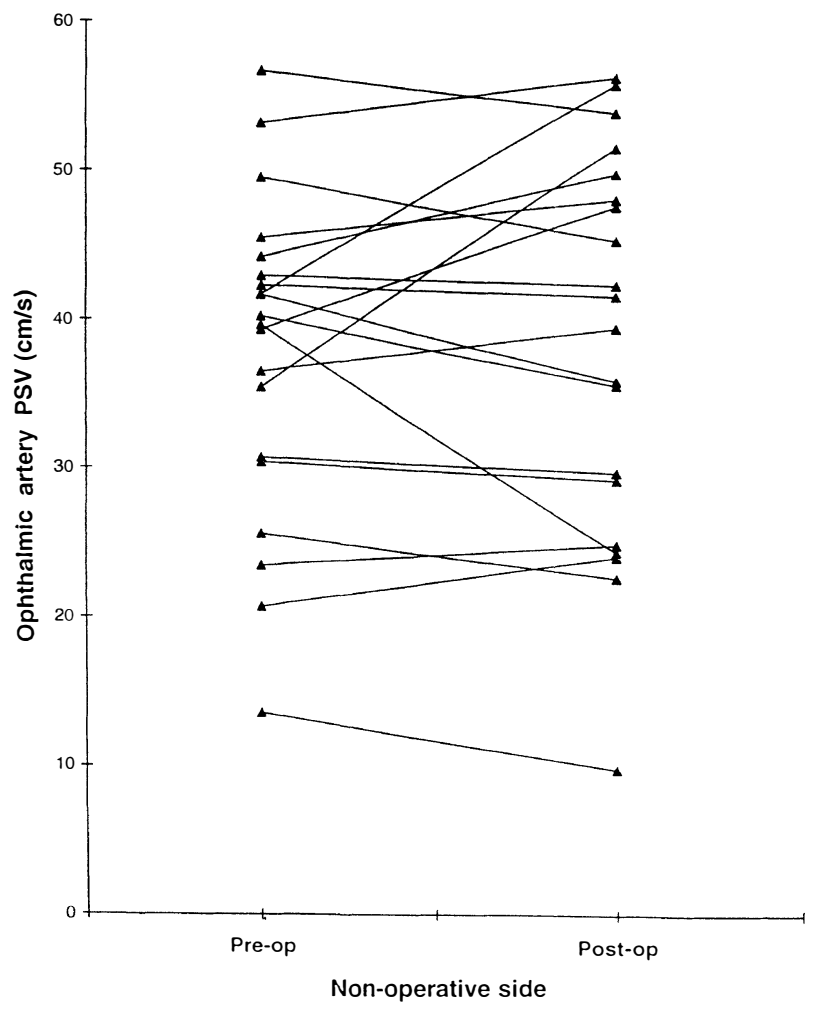

Fig. 4. Changes in PSV in the ophthalmic artery on the non-operated side before and after surgery.

necessarily imply a corresponding increase in arterial flow. The absence of any relationship with arterial velocities is inconsistent with the published results of two previous studies. One by Williamson and co-workers also obtained a negative correlation but with PSVs in the OA. ${ }^{17}$ At the same time, they found a positive

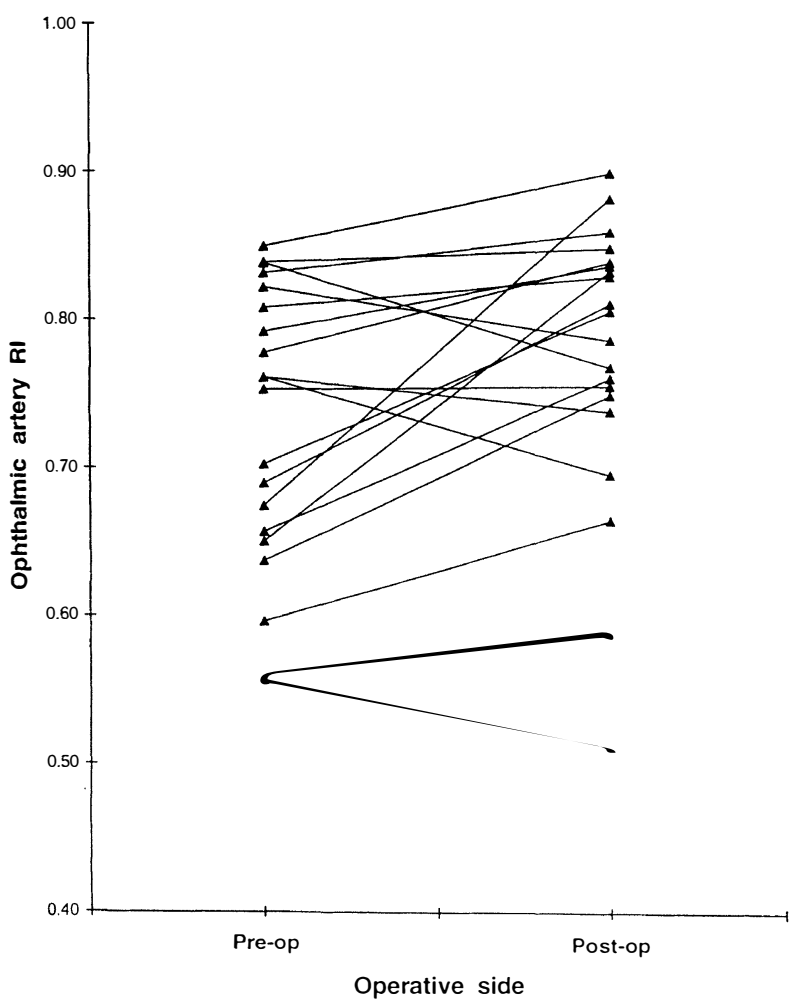

Fig. 5. Changes in resistive index (RI) of the ophthalmic artery on the operated side before and after surgery.

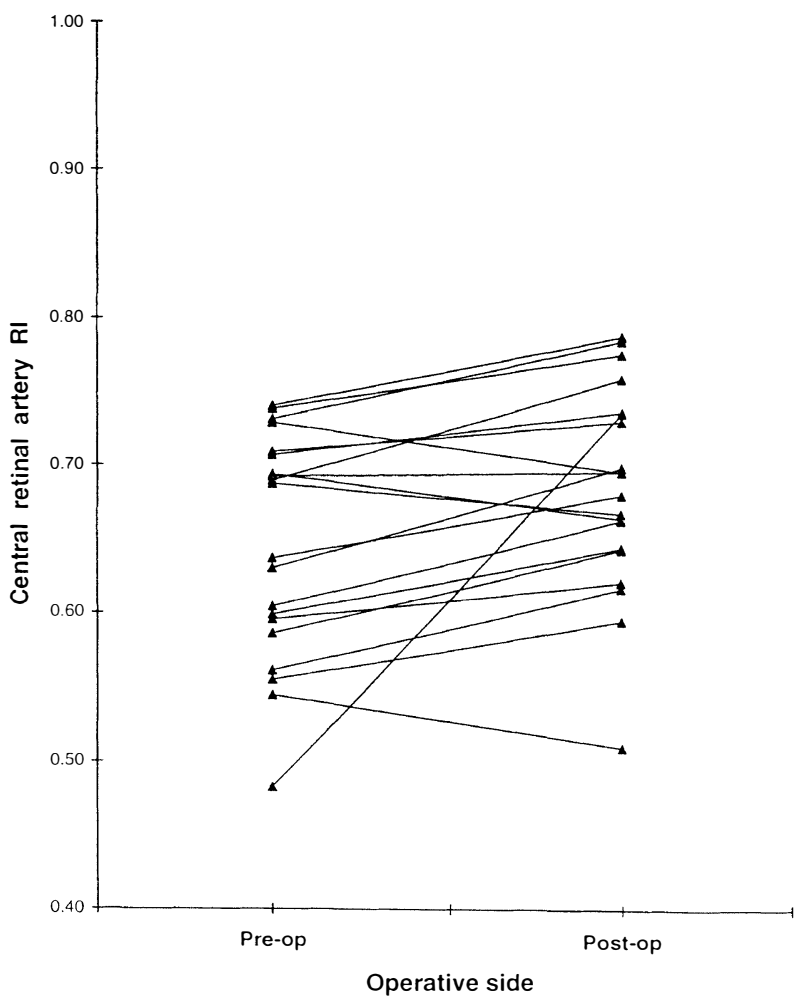

Fig. 6. Changes in RI of the central retinal artery on the operated side before and after surgery.

correlation with RI values in the same artery. A second study by Hu et al. ${ }^{11}$ discovered a positive correlation with systemic velocities in the CRA but not the OA. ${ }^{11}$ The above combination of findings underscores the fact that difficulty remains in the interpretation of such indirect measurements of the ophthalmic circulation. As pointed

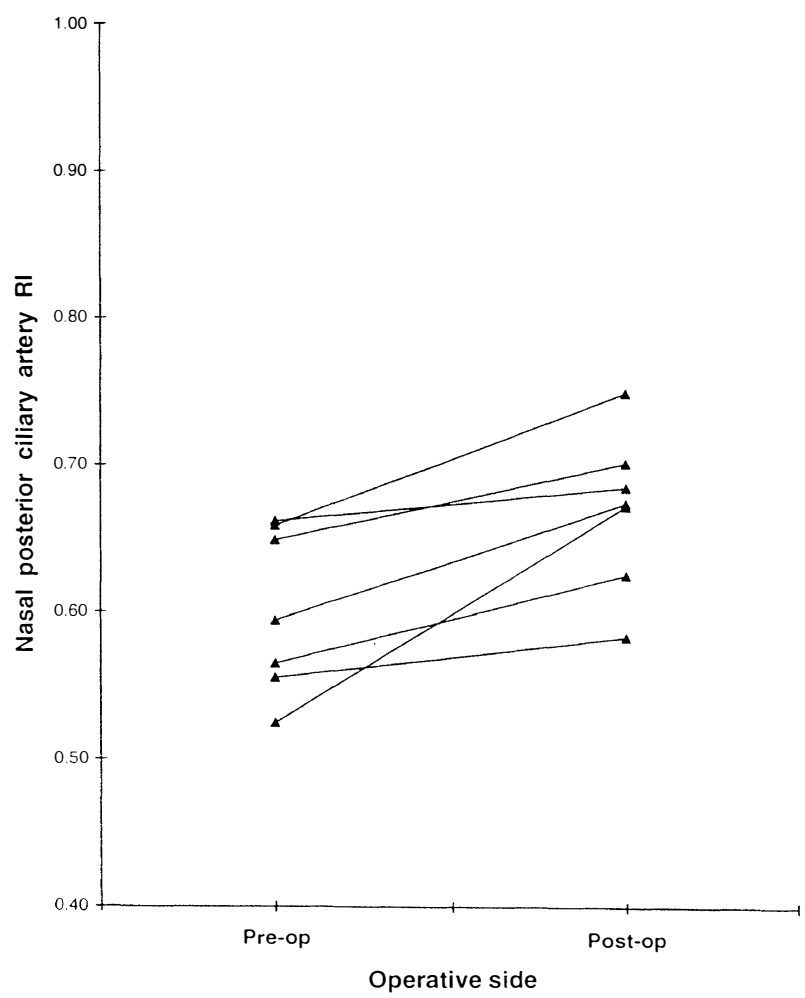

Fig. 7. Changes in RI of the nasal posterior ciliary artery on the operated side before and after surgery. 
Table 5. Median of ophthalmodynamometry measurements

\begin{tabular}{cccccc}
\hline & \multicolumn{2}{c}{ Operative side } & & \multicolumn{2}{c}{ Non-operative side } \\
\cline { 2 - 3 } \cline { 5 - 6 } & Pre-op. & Post-op. & & Pre-op. & Post-op. \\
\hline Median $(\mathrm{mmHg})$ & 42.0 & 45.0 & & 42.5 & 43.4 \\
\hline
\end{tabular}

There was no significant difference between the pre- and postoperative values.

out by Williamson, this may be secondary to inherent inaccuracies of the techniques utilised (despite good reproducibility, in our study, of

ophthalmodynamometry) or highly complex interrelationships between the haemodynamic parameters of flow, perfusion pressure and velocity.

Authors who have looked at patients with carotid stenosis and ocular ischaemia have demonstrated that carotid endarterectomy or bypass has resulted in improved visual symptoms and signs. ${ }^{18-20}$ This provides reasonable evidence that such surgery leads to increased blood flow to the ipsilateral eye. However, Sivalingam and associates, ${ }^{21}$ who reviewed case histories of 52 consecutive patients seen at the Wills Eye Hospital with the diagnosis of ocular ischaemic syndrome, were unable to demonstrate sustained visual benefit of carotid endarterectomy or superficial temporal artery-middle cerebral artery bypass. More recently Ward, Geroulakos, Mawn and their respective collaborators have focused on the effects of such surgery on the ophthalmic circulation using CDU. ${ }^{22-24}$ Our finding of a statistically significant elevation in the OA PSV but not the EDV agrees with that of Geroulakos et al. ${ }^{23}$ Ward et al., ${ }^{22}$ in their case reports of only three patients, noted a linear relationship between velocities in the OA, CRA and PCA, that is, as the OA velocities improved so did velocities in the CRA and NPCA. This is an observation not apparent in our work. Although there was a rise in the median values of both the CRA and NPCA, statistical significance was not achieved. Mawn et al. ${ }^{24}$ noted an increase from preoperative values of mean systolic values in the CRA and PCA, but these did not achieve statistical significance. No comment was made regarding the outcome of OA measurements.

Autoregulation may be responsible for maintaining these parameters at the stable levels that we have observed. It may be that there is no requirement for increased flow as only in one of these patients was there a question of chronic ocular ischaemia. The fact that the CRA diastolic perfusion pressure also remained statistically unchanged seems to support this idea. It is possible that perfusion via the CRA and NPCA, which only supply the globe, is more tightly regulated than flow in the OA. Velocity measurement in the CRV is thought to be a more reliable indicator of ocular perfusion because of its inability to autoregulate. ${ }^{7}$ If this is true, then the lack of significant alteration to the CRV velocities is also consistent with the above hypothesis. However, the study by Geroulakos et al. ${ }^{23}$ showed improvements in retinal arteriole PSV and macula photostress recovery times, which suggest that ocular blood flow is augmented by surgery. It would have been helpful to know the reproducibility of CDU when applied to the retinal arterioles. Using ocular pneumoplethysmography, Araki and co-workers ${ }^{25}$ demonstrated elevated retinal and choroidal pulse pressures post-operatively, which would also point to a possible increase in ocular perfusion. We must emphasise that the situation might be entirely different in patients with established hypotensive retinopathy or the ocular ischaemic syndrome (OIS). It seems likely that in these patients ocular volumetric blood flow does indeed increase with surgery, as evidenced by resolution of clinical features of OIS and the well-recognised postoperative elevation in intraocular pressure in some patients with rubeosis iridis that is thought to be secondary to hyperperfusion of the ciliary body. ${ }^{18-20}$ The effect of endarterectomy in this population merits further investigation by CDU.

Ho and associates ${ }^{26}$ have shown that subjects with OIS have reduced CRA and PCA PSV. Every patient in their series also had confirmed severe unilateral or bilateral atherosclerotic carotid disease. In our sample population (28 eyes), the mean NPCA PSV was similar to that of our healthy participants (20 eyes), who had a mean of $10.4 \mathrm{~cm} / \mathrm{s}$. Similarly, the mean CRA PSV was not reduced in our vasculopathic subjects. In that same study, Ho et al. noted that three OIS sufferers who experienced visual loss were the only subjects in whom neither the temporal nor nasal PCAs were detectable. On this basis, they suggested that PCA hypoperfusion was a major prognostic factor and might be useful in determining the most appropriate form of treatment. If carotid endarterectomy improves PCA blood flow, then these patients stand to benefit the most. On the other hand, those in whom the PCA PSV is preserved may not require surgery. In our sample of seven patients with paired pre- and post-operative values, there was no significant elevation of the NPCA PSV with surgery. However, one patient demonstrated a $75 \%$ and $70 \%$ increase on the operated and non-operated sides respectively. In another, the NPCA on the side of surgery that was not detectable before the procedure, was easily seen with normal PSV following surgery.

$\mathrm{Hu}$ and co-workers ${ }^{11}$ noted that RI values decreased (although not to a statistically significant degree) as the degree of carotid stenosis worsened. Our findings have shown that the converse is true, that is, relieving carotid stenosis actually results in an increase in the arterial RI on the operated side. This lends support to the suggestion that alterations in resistance upstream from the site of measurement do influence the RI. However, the CRV RI seems to be spared from this effect.

In conclusion, our findings suggest that reproducibility of ocular CDU in patients with arteriopathic disease is probably not as good as in healthy subjects studied by ourselves and at other centres. Carotid endarterectomy in patients without signs of pre-existing ocular ischaemia, causes an increase in the OA PSV and RI of the OA, CRA and NPCA. Further study is needed to assist in defining the category 
of patients with ophthalmic hypoperfusion secondary to carotid artery stenosis or occlusion who will benefit most from a surgical approach. The changes in RI suggest that it is determined not only by the downstream vascular bed but also by upstream events, and therefore interpreting changes in RI and its relationship with the ocular and retinal circulation is fraught with difficulty.

Special thanks go to Drs Barry Lansdell and Pat Lockie for loan of the ophthalmodynamometer and automated perimeter; to Dr Michael Coote for reviewing the perimetry results; and to $\mathrm{Mr}$ Russell Boustead, Mr Chris Kamusinski and Dr George Kiroff for invaluable technical advice.

\section{References}

1. Fisher M. Transient monocular blindness associated with hemiplegia. Arch Ophthalmol 1952;47:167-203.

2. Dugan JD Jr, Green WR. Ophthalmologic manifestations of carotid occlusive disease. Eye 1991;5:226-38.

3. Williamson TH, Harris A. Ocular blood flow measurement. Br J Ophthalmol 1994;78:939-45.

4. Erickson SJ, Hendrix LE, Massaro BM, Harris GJ, Lewandowski MR, Foley WD, Lawson TL. Color Doppler flow imaging of the normal and abnormal orbit. Radiology 1989;173:511-6.

5. Lieb WE, Cohen SM, Merton DA, Shields JA, Mitchell DG, Golberg BB. Color Doppler imaging of the eye and orbit. Arch Ophthalmol 1991;109:527-31.

6. Baxter GM, Williamson TH. Color Doppler imaging of the eye: normal ranges, reproducibility, and observer variation. J Ultrasound Med 1995;14:91-6.

7. Williams TH, Harris A. Color Doppler ultrasound imaging of the eye and orbit. Surv Ophthalmol 1996;40:255-67.

8. Aburn NS, Sergott RC. Orbital colour Doppler imaging. Eye 1993;7:639-47.

9. Harris A, Williamson TH, Martin B, Shoemaker JA, Sergott RC, Spaeth GL, et al. Test/retest reproducibility of color Doppler imaging assessment of blood flow velocity in orbital vessels. J Glaucoma 1995;4:281-6.

10. Lieb WE, Flaharty PM, Sergott RC, Medlock RD, Brown GC, Bosley $\mathrm{T}$, et al. Color Doppler imaging provides accurate assessment of orbital blood flow in occlusive carotid artery disease. Ophthalmology 1991;98:548-52.

11. Hu HH, Sheng WY, Yen MY, Lai ST, Teng MMH. Color Doppler imaging of orbital arteries for detection of carotid occlusive disease. Stroke 1993;24:1196-203.
12. Williamson TH, Lowe GDO, Baxter GM. Influence of age, systemic blood pressure, smoking, and blood viscosity on orbital blood velocities. Br J Ophthalmol 1995;79:17-22.

13. Greenfield DS, Heggerick PA, Hedges TR. Color Doppler imaging of normal orbital vasculature. Ophthalmology 1995;102:1598-605.

14. Gerraty RP, Scott M, Styles CB. Detection and grading of carotid stenosis by Duplex ultrasound: comparison with digital subtraction angiography. Presented as an abstract at the 40th annual general and scientific meeting of the Royal Australasian College of Radiologists, 1989.

15. Wollf E. The eyball. In: Warwick R, editor. Wollf's anatomy of the eye and orbit: comparative anatomy of the visual apparatus. Philadelphia: WB Saunders, 1976:90.

16. Williamson TH, Baxter GM, Dutton GN. Colour Doppler velocimetry of the arterial vasculature of the optic nerve head and orbit. Eye 1993;7:74-9.

17. Williamson TH, Baxter GM, Pyott A, Wykes W, Dutton GN. A comparison of colour Doppler imaging of orbital vessels and other methods of blood flow assessment. Graefes Arch Clin Exp Ophthalmol 1995;233:80-4.

18. Kearns TP, Younge BR, Piepgras DG. Resolution of venous stasis retinopathy after carotid artery bypass surgery. Mayo Clin Proc 1980;55:342-6.

19. Neupert JR, Brubaker RF, Kearns TP, Sundt TM. Rapid resolution of venous stasis retinopathy after carotid endarterectomy. Am J Ophthalmol 1976;81:600-2.

20. Rubin JR, McIntyre KM, Lukens MC, Plecha EJ, Bernhard VM. Carotid endarterectomy for chronic retinal ischemia. Surg Gynecol Obstet 1990;171:497-501.

21. Sivalingam A, Brown GC, Magargal LE. The ocular ischemic syndrome. III. Visual prognosis and the effect of treatment. Int Ophthalmol 1991;15:15-20.

22. Ward JB, Hedges TR, Heggerick PA. Reversible abnormalities in the ophthalmic arteries detected by color Doppler imaging. Ophthalmology 1995;102:1606-10.

23. Geroulakos G, Botchway LT, Pai V, Wilkinson AR, Galloway JM. Effect of carotid endarterectomy on the ocular circulation and on ocular symptoms unrelated to emboli. Eur J Vasc Endovasc Surg 1996;11:359-63.

24. Mawn LA, Hedges TR, Rand W, Heggerick PA. Orbital color Doppler imaging in carotid occlusive disease. Arch Ophthalmol 1997;115:492-6.

25. Araki CT, Babikian VL, Cantelmo NL, Johnson WC. Cerebrovascular hemodynamic changes associated with carotid endarterectomy. J Vasc Surg 1991;13:854-60.

26. Ho AC, Lieb WE, Flaharty PM, Sergott RC, Brown GC, Bosley TM, et al. Color Doppler imaging of the ocular ischaemic syndrome. Ophthalmology 1992;99:1453-62. 